\title{
Responsiveness to Resistance-Based Multimodal Exercise Among Men With Prostate Cancer Receiving Androgen Deprivation Therapy
}

\author{
Dennis R. Taaffe, PhD, DSc, MPH a,b,c; Robert U. Newton, PhDa,b,c,d; Nigel Spry, MBBS, PhDa, , ; \\ David J. Joseph, MBBS a,f,g; and Daniel A. Galvão, PhDa,b
}

\begin{abstract}
Background: Androgen deprivation therapy (ADT) in the management of prostate cancer ( $\mathrm{PCa}$ ) results in an array of adverse effects, and exercise is one strategy to counter treatment-related musculoskeletal toxicities. This study assessed the prevalence of exercise responsiveness in men with $\mathrm{PCa}$ undergoing ADT in terms of body composition, muscle strength, and physical function. Methods: Prospective analyses were performed in 152 men (aged 43-90 years) with $\mathrm{PCa}$ receiving ADT who were engaged in resistance exercise combined with aerobic or impact training for 3 to 6 months. Wholebody lean mass and fat mass (FM), trunk FM, and appendicular skeletal muscle were assessed with dual x-ray absorptiometry; upper and lower body muscle strength were assessed with the onerepetition maximum; and physical function was assessed with a battery of tests (6-m usual, fast, and backward walk; 400-m walk; repeated chair rise; stair climb). Results: Significant improvements were seen $(P<.01)$ in lean mass $(0.4 \pm 1.4 \mathrm{~kg}$ [range, -2.8 to $+4.1 \mathrm{~kg}])$, appendicular skeletal muscle $(0.2 \pm 0.8 \mathrm{~kg}[$ range, -1.9 to $+1.9 \mathrm{~kg}])$, and all measures of muscle strength (chest press, $2.9 \pm 5.8 \mathrm{~kg}$ [range, -12.5 to $+37.5 \mathrm{~kg}$ ]; leg press, $29.2 \pm 27.6 \mathrm{~kg}$ [range, -50.0 to $+140.0 \mathrm{~kg}$ ]) and physical function (from $-0.1 \pm 0.5 \mathrm{~s}$ [range, +1.3 to $-2.1 \mathrm{~s}$ ] for the $6-\mathrm{m}$ walk; to $-8.6 \pm 15.2 \mathrm{~s}$ [range, +25.2 to $-69.7 \mathrm{~s}$ ] for the $400-\mathrm{m}$ walk). An increase in FM was also noted $(0.6 \pm 1.8 \mathrm{~kg}$ [range, -3.6 to $+7.3 \mathrm{~kg}$ ]; $P<.01$ ). A total of 21 men did not exhibit a favorable response in at least one body composition component, 10 did not experience improved muscle strength, and 2 did not have improved physical function. However, all patients responded in at least one of the areas, and 120 (79\%) favorably responded in all 3 areas. Conclusions: Despite considerable heterogeneity, most men with $\mathrm{PCa}$ receiving ADT responded to resistance-based multimodal exercise, and therefore our findings indicate that this form of exercise can be confidently prescribed to produce beneficial effects during active treatment. J Natl Compr Canc Netw 2019;17(10):1211-1220
\end{abstract} doi: 10.6004/jnccn.2019.7311

${ }^{a}$ Exercise Medicine Research Institute, and ${ }^{b}$ School of Medical and Health Sciences, Edith Cowan University, Joondalup, Western Australia, Australia; ${ }^{c}$ School of Human Movement and Nutrition Sciences, University of Queensland, Brisbane, Queensland, Australia; Institute of Human Performance, University of Hong Kong, Hong Kong, China; and ' Genesis Cancer Care, Joondalup, fFaculty of Medicine, University of Western Australia, Nedlands, and ${ }^{9}$ Sir Charles Gairdner Hospital, Nedlands, Western Australia, Australia.

\section{Background}

Androgen deprivation therapy (ADT) is used extensively in the management of localized and advanced prostate cancer $(\mathrm{PCa}){ }^{1}$ Although effective in delaying disease progression and enhancing survival, ${ }^{1,2} \mathrm{ADT}$ is associated with an array of adverse effects, including reduced muscle ${ }^{3}$ and bone mass, ${ }^{4}$ an increase in body fat, ${ }^{4}$ and reduced muscle strength and physical function, ${ }^{5}$ leading to an increased risk of comorbidities that compromise functioning and quality of life. ${ }^{6,7}$

One strategy to combat a number of these treatmentrelated toxicities is exercise; specifically, resistance exercise as a sole training mode or as part of a multimodal exercise program. We and others have demonstrated the efficacy of exercise programs containing a resistancebased component for improving muscle mass and strength, ${ }^{8-10}$ bone mass, ${ }^{11}$ and physical function, ${ }^{9}$ and reducing adverse effects such as fatigue ${ }^{12}$ and sexual health ${ }^{13}$ in men with PCa receiving ADT. Although study conclusions are that exercise is an effective countermeasure to the adverse effects of ADT on body composition, strength, and function, it is clear that considerable interindividual variation exists given the measures of variability provided, such as the standard deviation, and therefore some men may not derive a favorable response. In contrast, exercise interventions to mitigate the effects of ADT on whole-body and trunk fat mass (FM) have been less successful, ${ }^{10,14}$ although, given the variation in response as indicated by measures of variability, some men would respond favorably.

In recommending and prescribing exercise for patients, the referring clinician and the exercise professional administering the program must be confident that the desired beneficial effect will be achieved, and patients need to have an appreciation of the likelihood of a favorable response. This raises some questions. Do all patients who are prescribed and undertake exercise achieve the outcomes of interest ("responders"), and if not, what percentage is likely to not achieve the objectives 
("nonresponders"), and is this nonresponse related to factors such as age, body size, training duration, session attendance, or whether they were already receiving or had just commenced an ADT regimen? To address these questions, our study assessed the prevalence of exercise responsiveness among men with $\mathrm{PCa}$ receiving ADT in terms of body composition, muscle strength, and physical function.

\section{Methods}

\section{Patients}

This study examined 152 men with a histologic diagnosis of PCa and treated with ADT from 4 randomized exercise trials ${ }^{10-12,15}$ performed at the Exercise Medicine Research Institute at Edith Cowan University in Perth, Australia. Participants had complete data available on all outcome measures, and were assigned to exercise arms in 3 of the trials ${ }^{10,12,15}$ and the immediate exercise arm in the fourth trial ${ }^{11}$; 99 patients were receiving established ADT regimens that specified a minimum exposure of 2 months, ${ }^{10,12}$ and 53 were commencing ADT. ${ }^{11,15} \mathrm{~A}$ total of 52 men engaged in exercise training for 3 months, ${ }^{10,15}$ and 100 men engaged in exercise training for 6 months. ${ }^{11,12}$ Exclusion criteria included established metastatic disease; participation in structured exercise in the previous 3 months; inability to walk $400 \mathrm{~m}$; acute illness; or any musculoskeletal, cardiovascular, or neurologic disorder that could inhibit participants or put them at risk from exercising as determined by their physician. The studies were approved by the Human Research Ethics Committee of Edith Cowan University, and all patients provided written informed consent.

\section{Exercise Interventions}

Exercise training was undertaken $2^{10,12,15}$ or 3 times ${ }^{11}$ per week for approximately 60 minutes per session, conducted in small groups of up to 10 participants, and supervised. Two exercise trials used a combined resistance and aerobic exercise program ${ }^{10,15}$; one trial had 2 exercise arms that used either combined resistance and aerobic exercise or combined resistance and impactloading exercise ${ }^{12}$; and one trial used combined resistance, aerobic, and impact-loading exercise. ${ }^{11}$ Detailed descriptions of the exercise programs and their progression have been published elsewhere. ${ }^{10-12,15}$ Briefly, resistance exercise comprised training for the major upper and lower body muscle groups, with patients performing 2 to 4 sets of each exercise at an intensity of 6 to 12 repetition maximum (ie, the heaviest weight that can be lifted 6-12 times). Aerobic exercise consisted of various modes and included walking or jogging on a treadmill and cycling or rowing on a stationary ergometer at an intensity of $60 \%$ to $85 \%$ of estimated maximum heart rate for 15 to 40 minutes. Impact-loading exercise consisted of a series of bounding, hopping, skipping, leaping, and drop jumping activities. All sessions commenced with a warmup comprising low-level aerobic activities and concluded with a cooldown of stretching activities.

\section{Body Composition}

Whole-body lean mass (LM) and FM, trunk FM (TrFM), and appendicular skeletal muscle (ASM) were derived from a whole-body dual x-ray absorptiometry scan (Hologic Discovery A; Hologic, Inc.). ASM was calculated as the sum of upper limb and lower limb bone-free LM. ${ }^{16}$

\section{Muscle Strength and Physical Function}

Dynamic muscle strength for the upper and lower body was determined using the 1-repetition maximum ${ }^{17}$ for the chest press and leg press exercises, respectively. Physical function was assessed using a battery of tests ${ }^{9,10,18}$ that included usual and fast 6-m walk (as measures of gait speed); 6-m backward tandem walk (as a measure of dynamic balance); 400-m walk (as a measure of cardiorespiratory fitness and walking endurance); stair climb (as a measure of lower body muscle power); and repeated chair rise, which measures the time needed to rise from a chair 5 times (as a measure of lower body function).

\section{Other Measures}

Demographic and clinical data were collected through self-report and medical records, respectively. Height and weight were assessed using a stadiometer and electronic scales, respectively, with body mass index (BMI; $\mathrm{kg} / \mathrm{m}^{2}$ ) calculated. Testosterone and prostate-specific antigen were measured at a commercial laboratory (PathWest Laboratory Medicine).

\section{Statistical Analyses}

Data were analyzed using SPSS Statistics, version 24 (IBM Corp). Normality of the distribution was assessed using the Kolmogorov-Smirnov test. Descriptive statistics included the mean and standard deviation and the minimum and maximum values. Pre- and posttraining changes were determined using either paired $t$ tests or the Wilcoxon signed rank test, as appropriate. Between-group differences for 3 versus 6 months were determined using independent $t$ tests or the MannWhitney $U$ test. Comparisons between responders and nonresponders regarding age, BMI, commencing versus receiving established ADT regimens, duration of exercise, and exercise attendance (with good attendance categorized as $\geq 70 \%$ of sessions) were performed using independent $t$ tests or the Mann-Whitney $U$ and 
chi-square tests. A responder was defined as experiencing improvement and a nonresponder as experiencing no improvement in the outcome measure of interest. ${ }^{19-22}$ Tests were 2-tailed, and to adjust for the number of comparisons, statistical significance was set at $\alpha=0.01$.

\section{Results}

Demographic and clinical characteristics of the study population are shown in Table 1. Participants were aged 43 to 90 years, had a BMI of 19.1 to $38.9 \mathrm{~kg} / \mathrm{m}^{2}$, and were predominantly married, nonsmokers, and no longer employed. Body composition, muscle strength, and physical function values before training are shown in Table 2.

After 3 to 6 months of exercise training, significant increases $(P<.01)$ were seen in LM $(0.4 \pm 1.4 \mathrm{~kg})$, ASM $(0.2 \pm 0.8 \mathrm{~kg})$, and FM $(0.6 \pm 1.8 \mathrm{~kg})$ (Table 3$)$. However, the response was heterogeneous with a wide variation, with minimum and maximum values of -2.8 to $+4.1 \mathrm{~kg},-1.9$ to $+1.9 \mathrm{~kg}$, and -3.6 to $+7.3 \mathrm{~kg}$ for LM, ASM, and FM, respectively. No significant change in TrFM was seen $(P=.346)$; however, the variation was wide $(-2.8 \mathrm{~kg}$ to $+4.7 \mathrm{~kg})$. Similarly, a significant increase $(P<.001)$ occurred in chest press and leg press strength that equated to gains of $9.5 \% \pm 18.2 \%$ and $30.4 \% \pm 34.9 \%$, respectively, with a wide variation in response. All physical function measures improved $(P<.01)$, with time to perform the task reduced. The largest absolute change was for the $400-\mathrm{m}$ walk $(-8.6 \pm 15.2 \mathrm{~s}$; range, +25.2 to $-69.7 \mathrm{~s}$ ), and the largest relative change was for the chair rise test $(-7.3 \% \pm 11.4 \%$; range, $+27.6 \%$ to $-40.7 \%$ ).

Changes by duration of training and ADT status are shown for body composition in Table 4 and for muscle strength and physical function in Table 5. Overall, for duration of training, the only significant difference was in FM $(P<.001)$, with an increase of $0.9 \pm 1.9 \mathrm{~kg}$ in the 6 -month group compared with $-0.1 \pm 1.3 \mathrm{~kg}$ in the 3 -month group. There was no significant difference in training duration among those receiving established $\mathrm{ADT}$ regimens; however, in those commencing ADT, there was a significant difference in FM $(P=.002)$, with an increase of $1.3 \pm 1.8 \mathrm{~kg}$ in the 6-month group compared with $-0.2 \pm 1.3 \mathrm{~kg}$ in the 3 -month group.

Figures 1-3 show the absolute change in body composition; muscle strength, chair rise, and stair climb performance; and physical function (ie, walking tasks), respectively, for each individual. The number of patients who showed an increase in LM or ASM greatly exceeded the number who showed decline (112 responders vs 40 nonresponders), whereas slightly more individuals showed increased rather than reduced FM or TrFM (78 nonresponders vs 74 responders), with a larger magnitude of change. Most men showed improvement in chest press (101 responders

Table 1. Participant Characteristics

\begin{tabular}{|c|c|c|c|}
\hline Characteristic & $\begin{array}{c}\text { 3-Month } \\
\text { Exercise Analysis } \\
(n=52)\end{array}$ & $\begin{array}{l}\text { 6-Month } \\
\text { Exercise Analysis } \\
(n=100)\end{array}$ & $\begin{array}{c}\text { All } \\
(N=152)\end{array}$ \\
\hline Age, mean (SD), y & $69.1(7.2)$ & $68.7(8.1)$ & $68.8(7.7)$ \\
\hline Height, mean (SD), cm & $172.1(6.1)$ & $172.9(6.0)$ & $172.6(6.1)$ \\
\hline Weight, mean (SD), kg & $83.0(11.6)$ & $82.7(14.0)$ & $82.8(13.2)$ \\
\hline $\mathrm{BMI}$, mean (SD), $\mathrm{kg} / \mathrm{m}^{2}$ & $28.0(3.7)$ & $27.5(3.7)$ & $27.7(3.7)$ \\
\hline ADT duration, median (IQR), mo & $2.0(0.0-5.0)$ & $2.0(0.0-3.0)$ & $2.0(0.0-3.0)$ \\
\hline Married, n (\%) & $44(84.6)$ & $79(80.6)$ & $123(82.0)$ \\
\hline Currently employed, $\mathrm{n}(\%)^{\mathrm{a}}$ & $12(23.1)$ & $34(34.7)$ & $46(30.7)$ \\
\hline Tertiary education, n (\%) & $17(32.7)$ & $43(43.0)$ & $60(39.5)$ \\
\hline Current smoker, $\mathrm{n}(\%)^{\mathrm{a}}$ & $2(3.8)$ & $5(5.1)$ & $7(4.6)$ \\
\hline Prostatectomy, n (\%) & $16(40.0)$ & $34(34.3)$ & $50(36.0)$ \\
\hline Radiation, n (\%) & $11(39.3)$ & $68(68.0)$ & $79(61.7)$ \\
\hline Number of comorbidities, median (IQR) & $1.0(0.0-2.0)$ & $0.0(0.0-1.0)$ & $1.0(0.0-2.0)$ \\
\hline
\end{tabular}

Abbreviations: ADT, androgen deprivation therapy; BMI, body mass index; PSA, prostate-specific antigen; IQR, interquartile range.

aMissing values: married, $n=2$; currently employed, $n=2$; current smoker, $n=1$; prostatectomy, $n=13$; radiation, $n=24$.

${ }^{\mathrm{b}}$ Cardiovascular disease, hypertension, dyslipidemia, diabetes. 


\begin{tabular}{|c|c|c|c|}
\hline & Mean (SD) & Minimum & Maximum \\
\hline \multicolumn{4}{|l|}{ Body composition, kg } \\
\hline Lean mass & $56.8(7.8)$ & 35.8 & 76.8 \\
\hline Fat mass & $23.5(7.1)$ & 9.7 & 49.9 \\
\hline Trunk fat mass & $12.7(4.5)$ & 3.7 & 29.0 \\
\hline ASM & $24.3(3.7)$ & 13.5 & 34.1 \\
\hline \multicolumn{4}{|l|}{ Muscle strength, $\mathrm{kg}$} \\
\hline Chest press & $39.7(12.3)$ & 10.0 & 70.0 \\
\hline Leg press & $125.3(50.2)$ & 20.0 & 330.0 \\
\hline \multicolumn{4}{|l|}{ Physical function, s } \\
\hline 6-m usual walk & $4.6(0.8)$ & 3.2 & 7.8 \\
\hline 6-m fast walk & $3.4(0.5)$ & 2.3 & 5.7 \\
\hline 6-m backward walk & $17.4(7.7)$ & 5.8 & 69.3 \\
\hline 400-m walk & $256.6(38.2)$ & 191.0 & 470.7 \\
\hline Chair rise & $12.0(2.4)$ & 8.0 & 20.1 \\
\hline Stair climb & $4.9(1.2)$ & 3.0 & 10.4 \\
\hline
\end{tabular}

Abbreviation: ASM, appendicular skeletal muscle.

vs 51 nonresponders) and leg press (127 responders vs 25 nonresponders) strength, with modest changes for nonresponders. More patients improved in the various physical function tasks than had no change or declined (6-m usual walk, 84 vs 68 patients; 6 -m fast walk, 89 vs 63 patients; 6 -m backwards walk, 108 vs 44 patients; 400 -m walk, 109 vs 43 patients; chair rise, 120 vs 32 patients; stair climb, 83 vs 69 patients), with a greater magnitude of change.

Overall, 20 patients (13\%) did not have a favorable response in at least one body composition component; $9(6 \%)$ did not improve in a measure of muscle strength; $1(<1 \%)$ did not improve in body composition and strength; and $2(1 \%)$ did not improve in any measure of physical function. For body composition, nonresponders tended to be older $(P=.023)$ and engaged in exercise training for 6 months $(P=.010)$, with no difference in BMI $(P=.283)$, exercise attendance $(P=.885)$, or commencing versus receiving established ADT regimens $(P=.408)$. Similarly, there was no difference in age $(P=.940)$, BMI $(P=.905)$, exercise attendance $(P=.216)$, or commencing versus receiving established ADT regimens $(P=.738)$ between responders and nonresponders regarding muscle strength, although they tended to have trained for 6 months $(P=.095)$. The 2 men who did not respond in physical function were commencing ADT, underwent 6 months of training with good attendance, and had BMIs of 22.4 and $29.8 \mathrm{~kg} / \mathrm{m}^{2}$ and were aged 79 and 68 years, respectively. Consequently, all patients responded in at least one measure of body composition, muscle strength, and physical function, and $79 \%$ had a positive response in some component of all 3 assessment areas.

\section{Table 3. Absolute and Relative Changes After 3 to 6 Months of Training}

\begin{tabular}{|c|c|c|c|c|c|c|}
\hline & \multicolumn{3}{|c|}{ Absolute } & \multicolumn{3}{|c|}{ Relative (\%) } \\
\hline & Mean (SD) & Minimum & Maximum & Mean (SD) & Minimum & Maximum \\
\hline \multicolumn{7}{|l|}{ Body composition, kg } \\
\hline Lean mass & $0.4(1.4)^{a}$ & -2.8 & +4.1 & $0.7(2.5)$ & -5.1 & +9.0 \\
\hline Fat mass & $0.6(1.8)^{a, b}$ & -3.6 & +7.3 & $3.1(8.5)$ & -19.3 & +28.9 \\
\hline Trunk fat mass & $0.1(1.2)$ & -2.8 & +4.7 & $1.4(10.3)$ & -27.4 & +39.5 \\
\hline ASM & $0.2(0.8)^{c}$ & -1.9 & +1.9 & $1.1(3.2)$ & -5.7 & +12.1 \\
\hline \multicolumn{7}{|l|}{ Muscle strength, kg } \\
\hline Chest press & $2.9(5.8)^{b, c}$ & -12.5 & +37.5 & $9.5(18.2)$ & -21.7 & +125.0 \\
\hline Leg press & $29.2(27.6)^{\mathrm{b}, \mathrm{c}}$ & -50.0 & +140.0 & $30.4(34.9)$ & -25.0 & +211.2 \\
\hline \multicolumn{7}{|l|}{ Physical function, ${ }^{d} \mathrm{~s}$} \\
\hline 6-m usual walk & $-0.1(0.5)^{\mathrm{a}, \mathrm{b}}$ & +1.3 & -2.1 & $-2.3(11.0)$ & +32.6 & -46.0 \\
\hline 6-m fast walk & $-0.2(0.4)^{c}$ & +1.2 & -1.6 & $-3.9(10.9)$ & +42.2 & -39.0 \\
\hline 6-m backward walk & $-2.0(4.7)^{\mathrm{b}, \mathrm{c}}$ & +9.5 & -26.4 & $-6.5(25.5)$ & +88.6 & -52.4 \\
\hline 400-m walk & $-8.6(15.2)^{b, c}$ & +25.2 & -69.7 & $-3.2(5.5)$ & +10.4 & -19.1 \\
\hline Chair rise & $-1.0(1.6)^{\mathrm{b}, \mathrm{c}}$ & +4.7 & -6.4 & $-7.3(11.4)$ & +27.6 & -40.7 \\
\hline Stair climb & $-0.1(0.5)^{a, b}$ & +2.2 & -2.0 & $-1.9(9.5)$ & +35.0 & -25.7 \\
\hline
\end{tabular}

Abbreviation: ASM, appendicular skeletal muscle.

a Significant change, $P<.01$.

bWilcoxon signed rank test.

'Significant change, $P<.001$.

dReduced time indicates improved performance from baseline to posttest. 


\section{Table 4. Absolute Change in Body Composition by Training Duration and ADT Status}

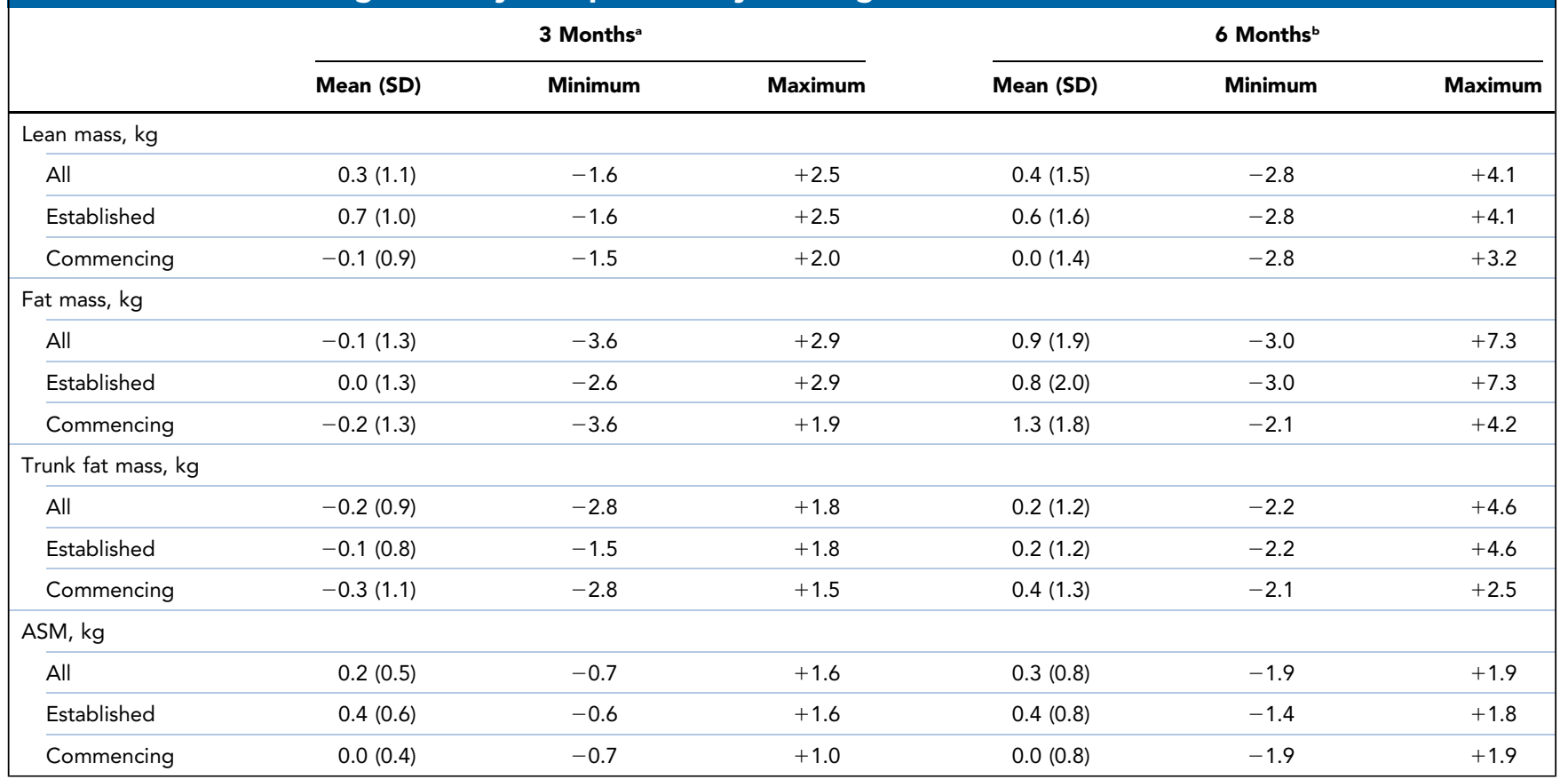

Abbreviations: ADT, androgen deprivation therapy; ASM, appendicular skeletal muscle.

aAl: $n=52$; established: $n=28$; commencing: $n=24$.

${ }^{b}$ All: $n=100$; established: $n=71$; commencing: $n=29$.

\section{Discussion}

This study examined exercise responsiveness among men with PCa receiving ADT in terms of body composition, muscle strength, and physical function (which are all adversely affected by ADT) to determine whether all patients prescribed and undertaking exercise achieve outcome objectives, and if not, what percentage are likely to be nonresponders. After either 3 or 6 months of supervised resistance-based multimodal exercise, $79 \%$ had a favorable response in at least one parameter of all 3 areas of body composition, muscle strength, and physical function, with the remainder responding in varying degrees in at least one of the areas. Therefore, despite ADT, our findings show that this form of targeted exercise can be confidently prescribed and undertaken to derive beneficial effects in most men during active treatment.

Despite group improvements in all outcome measures except FM and TrFM, considerable interindividual variability was seen. However, responders far outnumbered nonresponders in each of the other individual components or parameters, and the magnitude of change in responders was greater than in nonresponders, ranging in relative terms from $-5.1 \%$ to $+9.0 \%$ for LM and up to $-25.0 \%$ to $+211.2 \%$ for leg press strength. Increases in muscle strength were substantial for most participants, especially for the leg press, and are of clinical importance, given the association between muscle strength and mortality from all causes and cancer in men. ${ }^{23}$ In exercise trials, a wide variation in response is not unusual. For instance, Kohrt et $\mathrm{al}^{24}$ reported that after 9 to 12 months of aerobic exercise-based training in older men and women, maximal aerobic power increased by $24 \%$, with a range of $0 \%$ to $58 \%$, whereas Bouchard et $\mathrm{al}, 25$ in the HERITAGE Family Study, reported a range of $0 \%$ to $100 \%$ increase after 20 weeks of training. Similarly, Hubal et $\mathrm{al}^{26}$ found considerable heterogeneity in muscle strength and size after 12 weeks of resistance training in men and women aged 18 to 40 years, with changes in strength of $0 \%$ to $250 \%$ and in muscle area of $-2 \%$ to $+59 \%$. However, nonresponsiveness in one outcome or measure does not preclude responsiveness and adaptation in another, as evident in our results, and given the systemic nature of exercise affecting multiple organ systems, numerous unmeasured or undetermined benefits would result. ${ }^{27}$

Both muscle strength/physical function and LM are likely important in the setting of $\mathrm{ADT}$ for $\mathrm{PCa}$, although the level of importance of each component will depend on the patient's needs and physiologic and functional requirements. For example, in those with advanced disease, preserving physical function by improving muscle strength and maintaining activities of daily living could be viewed as a clinically meaningfully outcome. Similarly, for those experiencing substantial loss of LM and at risk for developing sarcopenia and metabolic 
Table 5. Absolute Changes in Muscle Strength and Physical Function by Training Duration and ADT Status

\begin{tabular}{|c|c|c|c|c|c|c|}
\hline & \multicolumn{3}{|c|}{3 Months $^{a}$} & \multicolumn{3}{|c|}{6 Months $^{b}$} \\
\hline & Mean (SD) & Minimum & Maximum & Mean (SD) & Minimum & Maximum \\
\hline \multicolumn{7}{|l|}{ Chest press, kg } \\
\hline All & $3.6(4.6)$ & -5.0 & +22.5 & $2.5(6.3)$ & -12.5 & +37.5 \\
\hline Established & $3.9(3.7)$ & -3.8 & +10.0 & $3.2(6.7)$ & -12.5 & +37.5 \\
\hline Commencing & $3.3(5.6)$ & -5.0 & +22.5 & $0.7(5.0)$ & -7.5 & +15.0 \\
\hline \multicolumn{7}{|l|}{ Leg press, kg } \\
\hline All & $33.5(24.9)$ & -20.0 & +100.0 & $27.0(28.8)$ & -50.0 & +140.0 \\
\hline Established & $37.5(20.6)$ & +10.0 & +90.0 & $28.8(28.7)$ & -50.0 & +140.0 \\
\hline Commencing & $28.9(28.8)$ & -20.0 & +100.0 & $22.5(29.1)$ & -40.0 & +80.0 \\
\hline \multicolumn{7}{|l|}{ 6-m usual walk, ${ }^{c}$ s } \\
\hline All & $-0.2(0.4)$ & +0.9 & -1.1 & $-0.1(0.6)$ & +1.3 & -2.1 \\
\hline Established & $-0.4(0.4)$ & +0.3 & -1.1 & $-0.2(0.6)$ & +1.3 & -2.1 \\
\hline Commencing & $0.0(0.4)$ & +0.9 & -0.9 & $0.1(0.3)$ & +1.0 & -0.4 \\
\hline \multicolumn{7}{|l|}{ 6-m fast walk, ${ }^{c} \mathrm{~s}$} \\
\hline All & $-0.2(0.3)$ & +0.5 & -1.3 & $-0.1(0.4)$ & +1.2 & -1.6 \\
\hline Established & $-0.3(0.4)$ & +0.5 & -1.3 & $-0.2(0.5)$ & +1.2 & -1.6 \\
\hline Commencing & $0.0(0.2)$ & +0.4 & -0.6 & $-0.1(0.2)$ & +0.3 & -0.6 \\
\hline \multicolumn{7}{|c|}{ 6-m backward walk, ${ }^{\mathrm{c}} \mathrm{s}$} \\
\hline All & $-2.3(5.2)$ & +7.2 & -26.4 & $-1.8(4.4)$ & +9.5 & -12.9 \\
\hline Established & $-5.0(5.3)$ & +0.3 & -26.4 & $-2.6(4.4)$ & +9.5 & -12.9 \\
\hline Commencing & $0.8(3.0)$ & +7.2 & -5.3 & $0.4(3.6)$ & +9.1 & -7.8 \\
\hline \multicolumn{7}{|l|}{ 400-m walk, ${ }^{c} \mathrm{~s}$} \\
\hline All & $-9.2(13.0)$ & +20.0 & -34.6 & $-8.3(16.3)$ & +25.2 & -69.7 \\
\hline Established & $-12.5(14.1)$ & +17.7 & -34.6 & $-10.2(17.7)$ & +22.9 & -69.7 \\
\hline Commencing & $-5.4(10.6)$ & +20.0 & -31.2 & $-3.6(11.2)$ & +25.2 & -28.3 \\
\hline \multicolumn{7}{|l|}{ Chair rise, ${ }^{c} \mathrm{~s}$} \\
\hline All & $-1.1(1.3)$ & +2.5 & -4.9 & $-0.9(1.7)$ & +4.7 & -6.4 \\
\hline Established & $-1.2(1.6)$ & +2.5 & -4.9 & $-1.0(1.9)$ & +4.7 & -6.4 \\
\hline Commencing & $-1.0(0.9)$ & +1.1 & -3.1 & $-0.6(1.0)$ & +1.7 & -2.9 \\
\hline \multicolumn{7}{|l|}{ Stair climb, , s } \\
\hline All & $-0.1(0.4)$ & +0.5 & -1.4 & $-0.1(0.6)$ & +2.2 & -2.0 \\
\hline Established & $-0.2(0.4)$ & +0.5 & -1.1 & $-0.1(0.7)$ & +2.2 & -2.0 \\
\hline Commencing & $-0.1(0.4)$ & +0.5 & -1.4 & $-0.2(0.4)$ & +0.3 & -1.9 \\
\hline
\end{tabular}

Abbreviation: ADT, androgen deprivation therapy.

aAll: $n=52$; established: $n=28$; commencing: $n=24$.

bAll: $n=100$; established: $n=71$; commencing: $n=29$.

'Reduced time indicates improved performance.

complications, preservation of LM will be critical. Conversely, in the older frail patient, enhancement in both parameters of lower limb muscle strength and LM may be required. As a result, individualization and the patient's clinical requirements will dictate the level of importance of the exercise response and required prescription to induce these adaptations.

For FM, the effects of exercise, if any, are modest when group values are analyzed in men receiving ADT, with no significant change generally reported, ${ }^{9,10,14}$ although this in itself is beneficial, given that FM increases with $\mathrm{ADT}^{4}$ and persists after treatment cessation. ${ }^{28} \mathrm{FM}$ (and TrFM) increased in just more than half of the men in this analysis, with the magnitude of increase greater than in those who experienced a reduction. However, nearly half of the participants did respond favorably, with reductions in whole-body FM $(\mathrm{n}=28$ with a reduction $>1 \mathrm{~kg})$ and $\operatorname{TrFM}(\mathrm{n}=25$ with a 
A

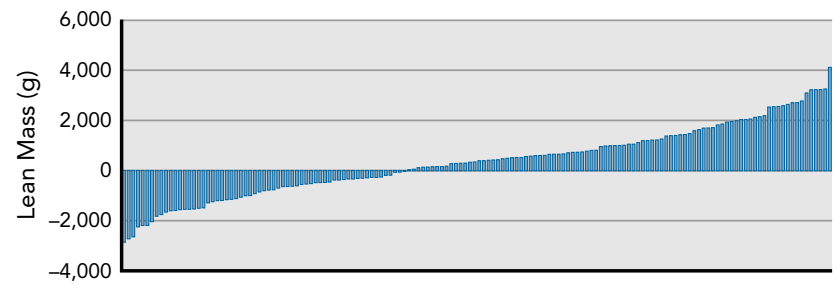

C

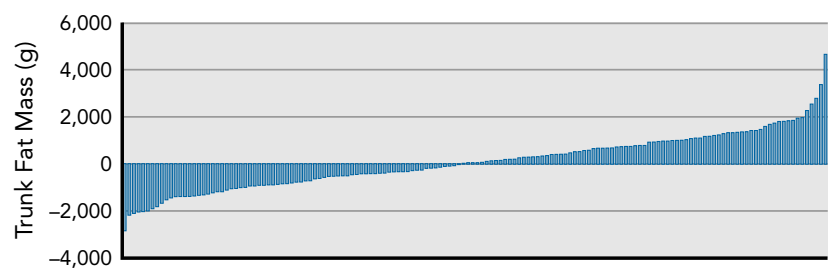

B

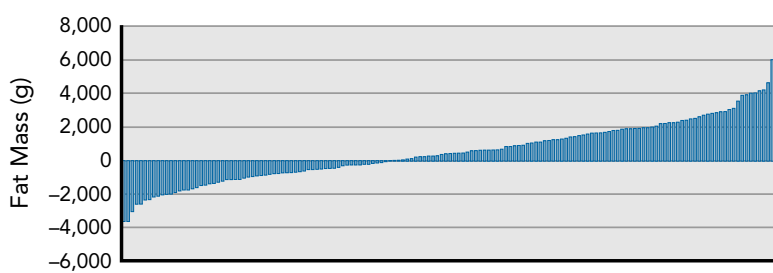

D

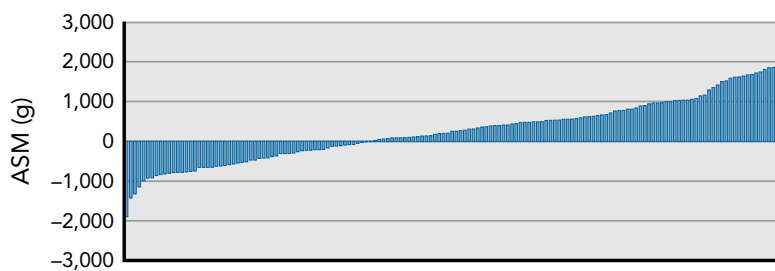

Figure 1. Absolute change in body composition for each individual: (A) lean mass, (B) fat mass, (C) trunk fat mass, and (D) appendicular skeletal muscle (ASM).

reduction $>1 \mathrm{~kg}$ ), and therefore the programs were successful for these patients and in some cases would be meaningful, given the magnitude of fat reduction. In a similar fashion, maximum oxygen consumption was directly assessed in a subgroup of men participating in one of the included trials, ${ }^{12}$ showing considerable variation in response (range, -8.8 to $+8.5 \mathrm{~mL} / \mathrm{kg} / \mathrm{min}$ ) such that a favorable response was observed in nearly half of the participants; therefore, for this outcome, as with FM and TrFM, a beneficial effect may be achieved for some men.

Importantly, every participant in the present study responded in at least one of the areas, with most showing favorable responses in body composition, strength, and physical function. Among nonresponders, no clear pattern was seen across the 3 outcome areas with regard to

\section{A}

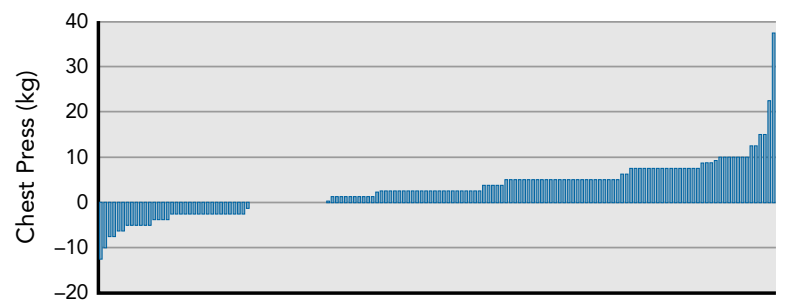

\section{C}

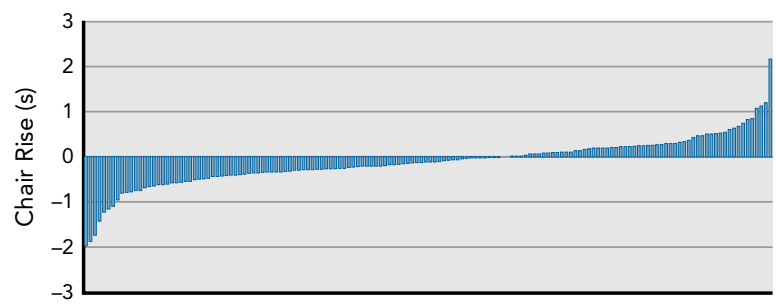

B

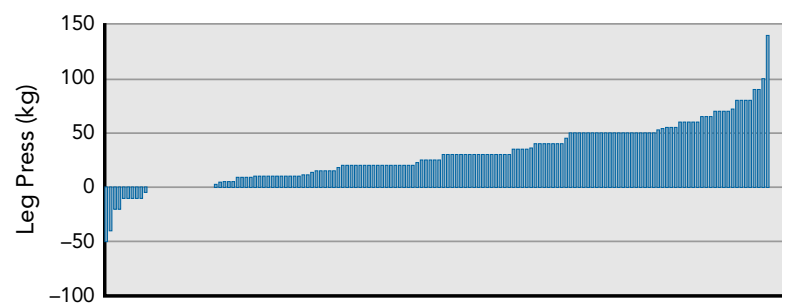

D

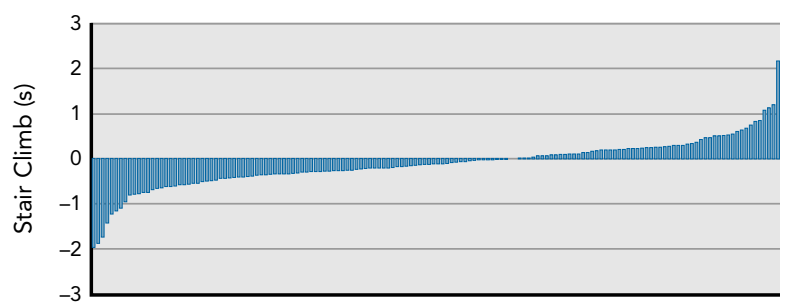

Figure 2. Absolute change in muscle strength, chair rise, and stair climb for each individual: (A) chest press, (B) leg press, (C) chair rise, and (D) stair climb. Reduced time for the chair rise and stair climb indicates improved performance. 
A

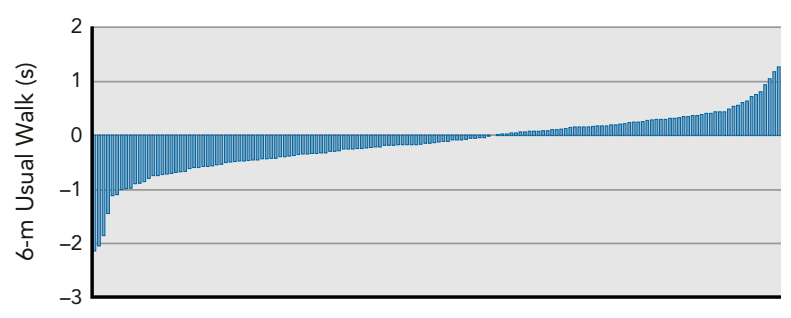

C

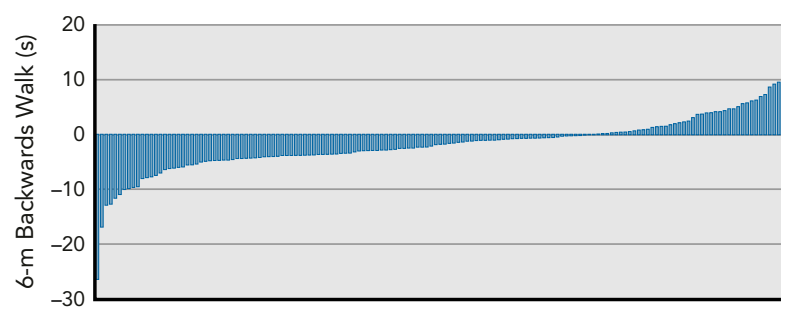

B

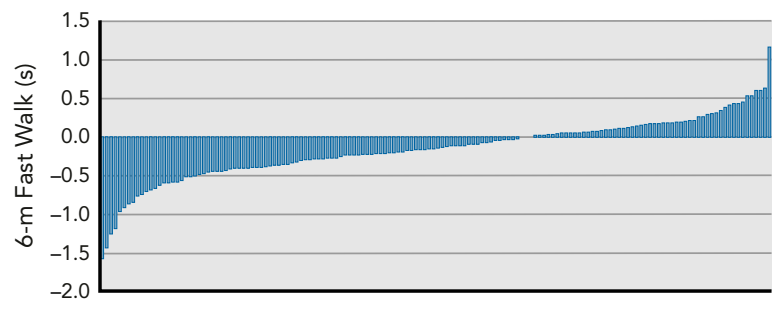

D

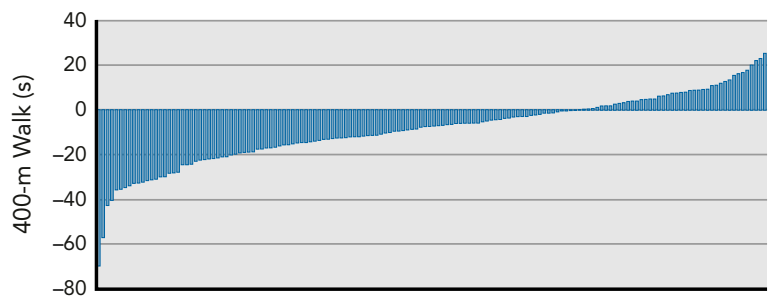

Figure 3. Absolute change in physical function for each individual: (A) 6-m usual walk, (B) 6-m fast walk, (C) 6-m backwards walk, and (D) 400-m walk. Reduced time for the walking tasks indicates improved performance.

age, BMI, commencing versus receiving established $\mathrm{ADT}$, or exercise attendance, although they were more likely to have engaged in exercise training for 6 months. It is not readily apparent what would account for this, because there was little difference in any baseline characteristic between those who trained for 3 versus 6 months. In any event, for nonresponders, given the dose-response nature of exercise on health-related outcomes, ${ }^{29,30}$ it may be that a higher exercise stimulus or dose would have resulted in a favorable response. For instance, Montero and Lundby ${ }^{31}$ recently showed that among healthy adults, all initially classed as nonresponders in cardiorespiratory fitness after 6 weeks of endurance training became responders after a successive 6-week training period that included additional weekly sessions. Similarly, Bonafiglia et al ${ }^{32}$ showed that the incidence of nonresponse in cardiorespiratory fitness parameters among men and women was reduced when the training stimulus was altered. Consequently, although multiple factors, both genetic and nongenetic, influence an individual's trainability, ${ }^{27}$ increased training time, volume, or intensity may be required in low-sensitivity individuals for a beneficial effect to occur. In addition, adjuvant therapies may be required to optimize the efficacy of exercise in these individuals, including nutritional strategies, such as protein supplementation and its timing in relation to exercise, ${ }^{33}$ and pharmacologic strategies. ${ }^{34,35}$ For instance, we recently determined that evidence supporting dietary interventions to mitigate adverse effects of ADT remains limited, ${ }^{36}$ and proposed a role for creatine supplementation to enhance exercise training adaptations in patients with cancer, including those with PCa receiving ADT. ${ }^{37}$

Given the applied prescription of the 4 trials included in our analyses, the resistance-based multimodal exercise program can be replicated in real-world practice. Protocols used in our trials followed initial recommendations from the American College of Sports Medicine, ${ }^{38}$ the American Cancer Society, ${ }^{39}$ and Exercise and Sports Science Australia, ${ }^{40}$ and were further expanded, prioritizing specificity of exercise prescription (eg, impact training to preserve bone loss). As a result, there would be opportunities for such exercise protocols to be incorporated into existing programs in the United States, such as LiveStrong at the YMCA, and globally. For example, from an Australian national program perspective, we have used similar prescriptions with positive results in a national community exercise program for men undergoing ADT for PCa. ${ }^{41}$ Moreover, it is never too late to introduce exercise; however, commencing exercise with the initiation of ADT may prevent the development of treatment-related toxicities. ${ }^{11}$

Strengths of this study include the reporting of data from 4 independent randomized controlled trials, with assessment of multiple body composition components using whole-body dual x-ray absorptiometry, and of physical function using a comprehensive battery of tests capturing different aspects of performance as well as upper and lower body dynamic muscle strength. As a 
result, we were able to assess the true utility of the exercise programs for improving physical functioning, which may have been missed had only 1 or 2 performance measures been assessed. In addition, the number of patients who underwent supervised training was relatively large, with complete data available for all outcome measures. We defined a responder as experiencing improvement and a nonresponder as experiencing no improvement in the outcome measure of interest. ${ }^{19-22}$ Although it may be argued that requiring a certain level of improvement is desirable to identify an individual as a responder, it should be noted that these men were undergoing ADT, and therefore in the absence of appropriate exercise, they would likely experience a loss of muscle mass and strength, a decline in physical function, and a gain in FM due to testosterone withdrawal. ${ }^{4}$ As a result, in this patient group, no change or minimal adverse change could be viewed as a beneficial outcome. Consequently, we believe that our definitions of responder and nonresponder are appropriate in this patient group.

\section{Conclusions}

In this group of men with PCa receiving ADT, most had a favorable response to supervised, resistance-based, multimodal exercise with regard to body composition, muscle strength, and physical function. Clinicians can be confident in referring a patient for exercise assessment and prescription, as can exercise physiologists and physical therapists designing and supervising the exercise programs, that most men with $\mathrm{PCa}$ receiving $\mathrm{ADT}$ will benefit from exercise with improved body composition and a higher level of strength and physical functioning.

Submitted October 4, 2018; accepted for publication April 12, 2019

Author contributions: Study concept and design: All authors. Data acquisition: All authors. Data analysis: Taaffe. Manuscript preparation: All authors. Manuscript approval: All authors.

Disclosures: The authors have not received any financial consideration from any person or organization to support the preparation, analysis, results or discussion of this article.

Correspondence: Dennis R. Taaffe, PhD, DSc, MPH, Exercise Medicine Research Institute, Edith Cowan University, 270 Joondalup Drive, Joondalup, WA 6027, Australia. Email: d.taaffe@ecu.edu.au

\section{References}

1. Parker C, Gillessen S, Heidenreich A, et al. Cancer of the prostate: ESMO clinical practice guidelines for diagnosis, treatment and follow-up. Ann Oncol 2015;26(Suppl 5):v69-77.

2. Keating NL, O'Malley AJ, McNaughton-Collins M, et al. Use of androgen deprivation therapy for metastatic prostate cancer in older men. BJU Int 2008;101:1077-1083

3. Smith MR, Saad F, Egerdie B, et al. Sarcopenia during androgendeprivation therapy for prostate cancer. J Clin Oncol 2012; 30:3271-3276.

4. Galvão DA, Spry NA, Taaffe DR, et al. Changes in muscle, fat and bone mass after 36 weeks of maximal androgen blockade for prostate cancer. BJU Int 2008;102:44-47.

5. Galvão DA, Taaffe DR, Spry N, et al. Reduced muscle strength and functional performance in men with prostate cancer undergoing androgen suppression: a comprehensive cross-sectional investigation. Prostate Cancer Prostatic Dis 2009;12:198-203.

6. Nguyen PL, Alibhai SM, Basaria S, et al. Adverse effects of androgen deprivation therapy and strategies to mitigate them. Eur Urol 2015; 67:825-836.

7. Spry NA, Kristjanson L, Hooton B, et al. Adverse effects to quality of life arising from treatment can recover with intermittent androgen suppression in men with prostate cancer. Eur J Cancer 2006;42:1083-1092.

8. Segal RJ, Reid RD, Courneya KS, et al. Resistance exercise in men receiving androgen deprivation therapy for prostate cancer. J Clin Oncol 2003;21:1653-1659.

9. Galvão DA, Nosaka K, Taaffe DR, et al. Resistance training and reduction of treatment side effects in prostate cancer patients. Med Sci Sports Exerc 2006;38:2045-2052.

10. Galvão DA, Taaffe DR, Spry N, et al. Combined resistance and aerobic exercise program reverses muscle loss in men undergoing androgen suppression therapy for prostate cancer without bone metastases: a randomized controlled trial. J Clin Oncol 2010;28:340-347.

11. Taaffe DR, Galvão DA, Spry N, et al. Immediate versus delayed exercise in men initiating androgen deprivation: effects on bone density and soft tissue composition. BJU Int 2019;123:261-269.

12. Taaffe DR, Newton RU, Spry N, et al. Effects of different exercise modalities on fatigue in prostate cancer patients undergoing androgen deprivation therapy: a year-long randomised controlled trial. Eur Urol 2017;72:293-299.

13. Cormie P, Newton RU, Taaffe DR, et al. Exercise maintains sexual activity in men undergoing androgen suppression for prostate cancer: a randomized controlled trial. Prostate Cancer Prostatic Dis 2013;16:170-175.
14. Segal RJ, Reid RD, Courneya KS, et al. Randomized controlled trial of resistance or aerobic exercise in men receiving radiation therapy for prostate cancer. J Clin Oncol 2009;27:344-351.

15. Cormie P, Galvão DA, Spry N, et al. Can supervised exercise prevent treatment toxicity in patients with prostate cancer initiating androgendeprivation therapy: a randomised controlled trial. BJU Int 2015;115: 256-266.

16. Heymsfield SB, Smith R, Aulet M, et al. Appendicular skeletal muscle mass: measurement by dual-photon absorptiometry. Am J Clin Nutr 1990; 52:214-218.

17. Taaffe DR, Duret $C$, Wheeler $S$, et al. Once-weekly resistance exercise improves muscle strength and neuromuscular performance in older adults. J Am Geriatr Soc 1999;47:1208-1214.

18. Galvão DA, Taaffe DR. Resistance exercise dosage in older adults: singleversus multiset effects on physical performance and body composition. J Am Geriatr Soc 2005;53:2090-2097.

19. Fisher G, Bickel CS, Hunter GR. Elevated circulating TNF- $\alpha$ in fat-free mass non-responders compared to responders following exercise training in older women. Biology (Basel) 2014;3:551-559.

20. Churchward-Venne TA, Tieland M, Verdijk LB, et al. There are no nonresponders to resistance-type exercise training in older men and women. J Am Med Dir Assoc 2015;16:400-411.

21. Böhm A, Weigert $C$, Staiger $H$, et al. Exercise and diabetes: relevance and causes for response variability. Endocrine 2016;51:390-401.

22. Corli O, Roberto A, Bennett MI, et al. Nonresponsiveness and susceptibility of opioid side effects related to cancer patients' clinical characteristics: a post-hoc analysis. Pain Pract 2018;18:748-757.

23. Ruiz JR, Sui X, Lobelo F, et al. Association between muscular strength and mortality in men: prospective cohort study. BMJ 2008;337:a439.

24. Kohrt WM, Malley MT, Coggan AR, et al. Effects of gender, age, and fitness level on response of VO2max to training in 60-71 yr olds. J Appl Physiol (1985) 1991;71:2004-2011.

25. Bouchard $C, A n P$, Rice $T$, et al. Familial aggregation of VO2max response to exercise training: results from the HERITAGE Family Study. J Appl Physiol (1985) 1999;87:1003-1008.

26. Hubal MJ, Gordish-Dressman H, Thompson PD, et al. Variability in muscle size and strength gain after unilateral resistance training. Med Sci Sports Exerc 2005;37:964-972.

27. Booth FW, Laye MJ. The future: genes, physical activity and health Acta Physiol (Oxf) 2010;199:549-556. 
28. Spry NA, Taaffe DR, England PJ, et al. Long-term effects of intermittent androgen suppression therapy on lean and fat mass: a 33-month prospective study. Prostate Cancer Prostatic Dis 2013;16:67-72.

29. Slentz CA, Duscha BD, Johnson JL, et al. Effects of the amount of exercise on body weight, body composition, and measures of central obesity: STRRIDE-a randomized controlled study. Arch Intern Med 2004;164:31-39.

30. Duscha BD, Slentz CA, Johnson JL, et al. Effects of exercise training amount and intensity on peak oxygen consumption in middle-age men and women at risk for cardiovascular disease. Chest 2005;128:2788-2793.

31. Montero D, Lundby $C$. Refuting the myth of non-response to exercise training: 'non-responders' do respond to higher dose of training. J Physiol 2017;595:3377-3387.

32. Bonafiglia JT, Rotundo MP, Whittall JP, et al. Inter-individual variability in the adaptive responses to endurance and sprint interval training: a randomized crossover study. PLoS One 2016;11:e0167790.

33. Esmarck $\mathrm{B}$, Andersen JL, Olsen $\mathrm{S}$, et al. Timing of postexercise protein intake is important for muscle hypertrophy with resistance training in elderly humans. J Physiol 2001;535:301-311.

34. Buford TW, Roberts MD, Church TS. Toward exercise as personalized medicine. Sports Med 2013;43:157-165.
35. Buford TW, Anton SD, Clark DJ, et al Optimizing the benefits of exercise on physical function in older adults. PM R 2014;6:528-543.

36. Barnes KA, Ball LE, Galvão DA, et al. Nutrition care guidelines for men with prostate cancer undergoing androgen deprivation therapy: do we have enough evidence? Prostate Cancer Prostatic Dis 2019;22:221-234

37. Fairman $\mathrm{CM}$, Kendall $\mathrm{KL}, \mathrm{Hart} \mathrm{NH}$, et al. The potential therapeutic effects of creatine supplementation on body composition and muscle function in cancer. Crit Rev Oncol Hematol 2019;133:46-57.

38. Schmitz KH, Courneya KS, Matthews C, et al. American College of Sports Medicine roundtable on exercise guidelines for cancer survivors. Med Sci Sports Exerc 2010;42:1409-1426.

39. Rock CL, Doyle C, Demark-Wahnefried W, et al. Nutrition and physical activity guidelines for cancer survivors. CA Cancer J Clin 2012;62: 243-274.

40. Hayes SC, Spence RR, Galvão DA, et al. Australian Association for Exercise and Sport Science position stand: optimising cancer outcomes through exercise. J Sci Med Sport 2009;12:428-434.

41. Beydoun N, Bucci JA, Chin YS, et al. Prospective study of exercise intervention in prostate cancer patients on androgen deprivation therapy. J Med Imaging Radiat Oncol 2014;58:369-376.

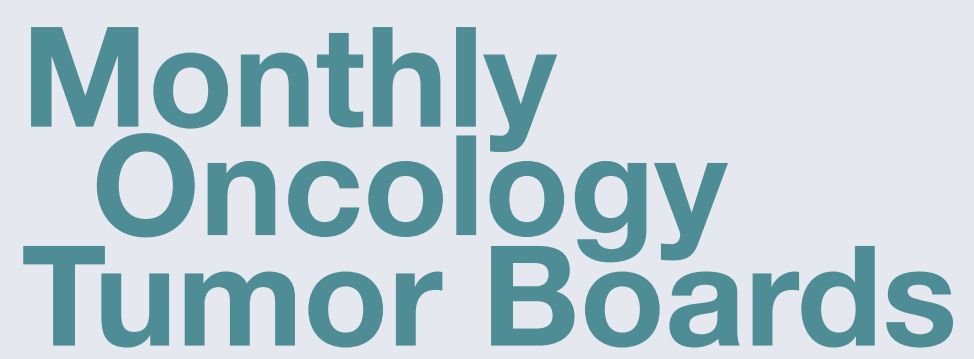

Emerging Strategies for Pediatric ALL Management

Monday, October 21, 2019 • 12:00 - 1:00 PM EDT

Andrea Mariani, MD and Nicole Bush, APRN, CNP Mayo Clinic Cancer Center

Visit education.nccn.org/tumorboards to register for upcoming webinars or to view previous presentations.

This activity is supported by an educational grant from AstraZeneca; Celgene Corporation; Clovis Oncology, Inc.; Eisai; Genomic Health, Inc.; Lilly; Novartis; Sanofi Genzyme; Taiho Oncology, Inc; and TESARO. This activity is supported by an independent medical education grant from Advanced Accelerator Applications, a Novartis company; Bristol-Myers Squibb; and Pharmacyclics LLC and Janssen. This activity is supported by an independent educational grant from AbbVie; Astellas and Pfizer, Inc.; and Merck \& Co., Inc. This activity is supported by a medical education grant from Exelixis, Inc. This activity is supported by an unrestricted educational grant from Gilead Sciences, Medical Affairs.

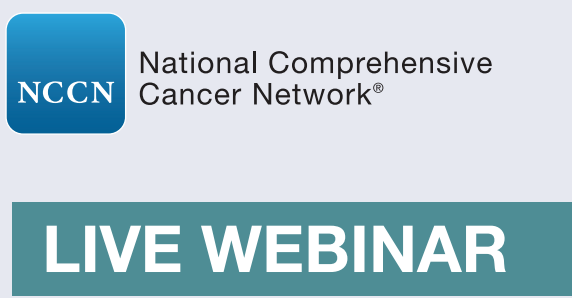

National Comprehensive

Cancer Network

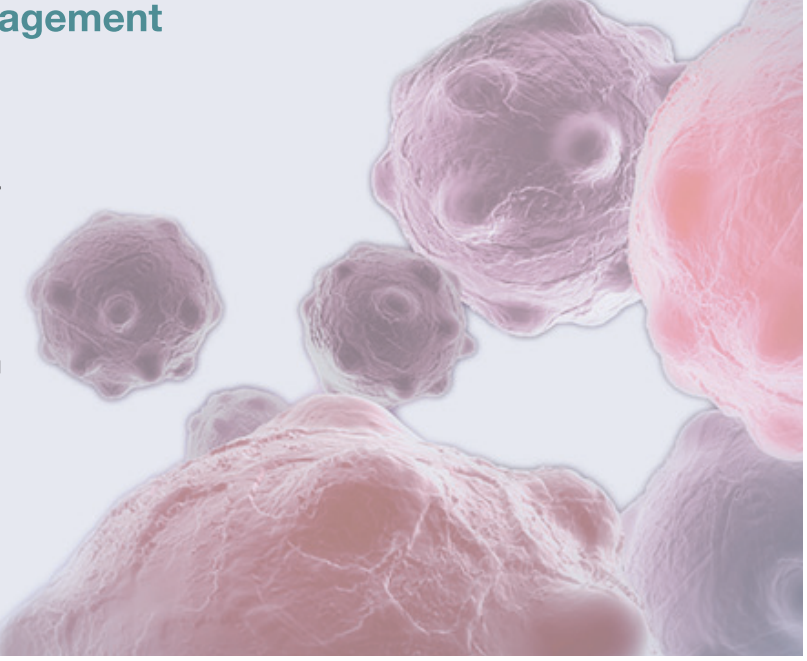

